\title{
The trade-off between DES and SD in modelling military manpower
}

\author{
Nethal K. Jajo*
}

Research Reporting, Analysis, Data and Systems, Research Portfolio, University of Sydney, Sydney, Australia

\begin{tabular}{l}
\hline C H R O N I C L E \\
\hline Article history: \\
Received October 28, 2014 \\
Received in revised format 2 \\
February 2015 \\
Accepted 4 February 2015 \\
Available online \\
February 42015 \\
\hline Keywords: \\
Discrete event simulation \\
Military morkforce \\
System dynamics and state of \\
space
\end{tabular}

A B S T R A C T

\begin{abstract}
Operations research techniques have been used widely in simulating the dynamics of workforce systems. Discrete Event Simulation (DES) and System Dynamics (SD) are among the techniques that have been increasingly used in modelling military workforces. In the last five years, DES has seen more interest in modelling both career management and the training pipeline. Two significant reasons for this are discussed in this paper. This article presents some notes in comparing the two techniques in modelling military workforce. The study found that DES is an appealing method in workforce modelling, especially with a small size population, as it more easily accommodates new personnel attributes and prevents the fractionalisation of personnel through the system.
\end{abstract}

(c) 2015 Growing Science Ltd. All rights reserved.

\section{Introduction}

The concept of modelling, on which simulation is based on, has been in use for many years. With the advent of computing technology, the application of simulation covers nearly every field of human endeavour. Simulation is the process of designing a computerised model of a system (or process) and conducting experiments with this model for the purpose either of understanding the behaviour of the system or of evaluating various strategies for the operation of the system (Shannon, 1975). Most military organisations have a large and complex workforce system which necessitates the use of simulation processes. It is critical that workforce planning is conducted in an integrated manner, ensuring optimal use of resources and maintaining the flexibility to adapt to current and future needs of both the military organisation and its nation. For a military organisation to be able to manage its manpower, it must be informed about its internal dynamics and the dynamics of its environment. Internal dynamics can be explored through personnel movements: promotions, internal transfers, redundancies and retirements. Influential external dynamics include the economic situation, legislation and policy changes.

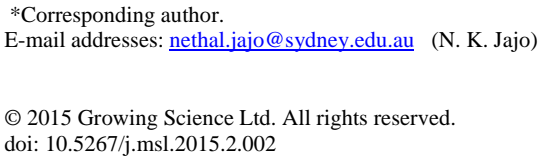


The interaction between individuals within a system is an important feature in determining the operations research tool suitable for modelling this system. If this interaction is significant then approaches such as Discrete Event Simulation and System Dynamics are required (Barton et al., 2004). Interaction between individuals is the main feature of the military manpower system. For example, interaction between individuals needs to be considered in modelling the 'pull promotion (a person is promoted if he or she is both qualified and needed to fill a vacancy at the next higher rank level)'; the probability of an individual being promoted to the next rank level depends on the number of personnel in the current level who are eligible for promotion and on the number of the vacant positions in the next rank level. The promotion of one personnel affects the numbers of personnel in the both rank levels and hence affects the promotion of other personnel. For the above mentioned reason and in agreement with others, DES and SD are the most suitable paradigms in modelling military workforce.

Over the past decade, there has been considerable interest in which approach should be used and when. Prior to 2005, most of the comparisons were biased as they were carried out either by DES or SD analysts (Morecroft \& Robinson, 2006) and fall short of answering the two mentioned questions. Since 2005, new comparison studies were conducted to compare the two approaches through collaborative research between expertise from DES and SD using empirical studies (Morecroft \& Robinson, 2005; 2006; Tako \& Robinson, 2009, 2010; Chahal \& Eldabi, 2010; Tanha et al., 2012), and a new approach began, combining both approaches in one model (Brailsford et al., 2010). Current empirical studies suggest that neither method is necessarily superior to the other, and that either method may be useful in a specific context.

This study presents notes comparing the appropriateness of the two systems for modelling military workforces. These notes concern both result outcomes and approach of each system.

In this study; $\eta(\mathrm{S})$ will represent the number of personnel within the set $\mathrm{S}$ and $\rho(\mathrm{S})$ represents the number of the nonempty and mutually exclusive subsets of the set $S$.

\section{Summary of DES and SD}

DES and SD represent certain features of the real world and both approaches also facilitate particular features of this world. The identified series of similarities and differences between DES and SD modelling through the mentioned references suggested neither approach is necessarily superior to the other, but that either method may be useful in a particular environment. In modelling a specified problem, the modellers need to be aware of the simplifications that may be provided by each approach and then select the modelling approach with features that match the features of the problem. More details about how to express models and their properties can be explored in Raczynski (2012). Following are the most known features of both DES and SD platforms.

\subsection{DES}

DES is an approach that represents individual entities that move through a series of queues and activities at discrete points in time. It is rooted back to the 1960s when Gordon (1961) conceived and evolved the idea for General Purpose Systems Simulator (GPSS) and brought about its IBM implementations. Entities (transactions in GPSS) are passive objects that represent people, parts, documents, tasks, messages. They travel through the blocks of the flowchart, where they stay in queues, are delayed and processed, seize and release resources, split and combine.

For historical review of DES development, see Robinson (2005) and Hollocks (2006) who reviewed in chronological order the history of DES for the period 1960-2005. DES models are in general stochastic models that make use of probability distributions and random sampling to allow for the uncertainty involved in events such as enlistments, separations and promotions. By combining stochastic models 
with repeated simulation, it is possible to determine the probability that certain outcomes will be achieved.

Many researches, among them Sweetser (1999), believe that DES models provide a detailed analysis of systems and produce an extensive statistically valid analysis of the historical behaviour of the systems under study. One of hundreds of DES platforms is the SIMAN process and simulation language. In 2000, Rockwell Automation (formerly System Modelling) used SIMAN process and simulation language to develop DES software named 'Arena'.

Arena is a Microsoft Windows operating system application and is fully compatible with other Windows software, like word processors, spreadsheets and CAD packages (Kelton et al., 2007). It is an entity based modelling package; that is, each entity in the model is treated individually. Arena is mainly discrete event simulation software and has the ability to accommodate both continuous and hyper systems. The selection of which system to apply is straightforward when building the model. This software provides graphical and numerical displays as well as computer animation of the system. It uses icons to represent individual staff members (entities) moving through the system. The animation of personnel flow and on-screen movement in an Arena model can be a valuable tool in providing increased understanding of the flow. Arena as the DES package has widely spread in use for military workforce and training pipeline modelling by many nations' organisations working in the area of strategic human resources and security (see Maryam et al., 2012).

\section{$2.2 S D$}

SD is an approach that applies concepts from engineering servomechanism theory to social science. It was developed in the late 1950s by Forrester (Forrester, 1961). In his early book, Forrester defines industrial dynamics as the study of information feedback characteristics of industrial activity to show how organisational structure, amplification (in policies) and time delays (in decisions and actions) interact to influence the success of the enterprise (Forrester, 1961). The use of stocks and flows are the main characteristic of the SD structure. In a workforce, stocks are the number of people in a particular class at a particular time and flows are the number of members moving from one class to another class in a given time interval.

SD technology was originally designed with support only for systems where states change in a continuous manner over time. An SD model typically aggregates discrete events of the underlying system to a level where the events can considered to be part of a continuous flow. Continuous simulations use integration with respect to time to represent the development of systems. Powersim studio (Powersim Software AS, 2002), as one of the SD platforms, is mainly continuous event simulation software and supports accumulation in addition to integration. The defining characteristic of SD modelling tools, such as Powersim, is the use of stock-and-flow diagrams, feedback loops and delays and the ability to use slide-bars, or similar functionality, to graphically demonstrate quickly and dynamically the results of varying inputs and make SD models a good fit with the workforce planning problems.

\section{Some important issues in modelling military workforce}

The real-world workforce of an organisation can be divided into subsets that are homogeneous according to attributes to be treated in the model. These subsets can be subdivided into smaller subsets based on age, length of service, time in position, location and experience, etc.

Military personnel can, for example, be classified according to their rank and occupation. A military workforce is characterised by its closed nature and strict hierarchy, that is: only recruiting externally 
into the lowest rank and filling vacancies at higher ranks internally by promotion. These characteristics make a military workforce distinct from any civilian organisations' workforces.

The DES model works at an individual level, allowing full representation of each individual's history and the interaction between specific individuals. Moreover DES models can accommodate very easily many new attributes that may be required and added to the model to adopt any future organisation policy changes.

In SD modelling, each classified rank (or occupation) is called a state. The set of all possible states defines the state-space of the model. A vector of stocks is used to represent the number of personnel in each state at a particular time. The flow covers all movements, both between states in the system and also to or from the outside. These include recruitment, voluntary wastage (labour turnover), involuntary wastage, promotion, internal transfer and aging. This makes SD models a good fit with the workforce planning problem. On other hand, this modelling approach maintains the state space which is problematic. The following two issues may be considered in modelling military workforce systems.

\subsection{Attributes tracking system and state space disintegration}

The richness and complexity of the military workforce system and the internal and external interaction between its components necessitate the use of many attributes in modelling the system. Modelling a military workforce involves forecasts of hundreds to tens of thousands of individuals who are characterised by multiple attributes and some of which have tens of sub attributes. Moreover, it is desirable to extend the model to accommodate merging issues of interest. This often requires adding new attributes to be tracked in the model.

In DES, these attributes can be assigned to each entity in a very simple and straightforward procedure. It simply requires stating the attributes in the definition of the entity in the model, along with any code desired to properly model the attributes. Unlimited attributes can be tagged to an entity representing different individual identities. The overall entities can be merged or streamed based on their attributes. In SD modelling, on the other hand, attributes tracking is extremly difficult. As a simple example, in modelling a military population, this population can be divided into states corresponding to each rank, with numbers in each state corresponding to how many individuals there are at each rank. The flow between the states would correspond to promotions from one rank to another. Assume the existence of just six ranks: Lieutenant (LT), Captain (CAPT), Major (MAJ), Lieutenant Colonel (LTCOL), Colonel (COL) and Brigadier (BRIG). To accommodate another attribute, say Time in Rank (TiR), each of these rank states would be subdivided into rank-TiR states (for example; CAPT with 0 year TiR, CAPT with 1 year TiR). If we assume, arbitrarily, the existence of just ten TiR values, the explicitly accommodating specific states in this range of values would require in the order of 151,200 (10 permutation 6) distinct states, which is tedious and clearly non-trivial. To accommodate another attribute, say Military Occupations (MO), each of these rank-TiR states would be subdivided into MO states. Increasing the number of the ranks and the number of military occupations to match the real life military numbers in both ranks and occupations, and even adding more attributes (Year in Service (YiS), qualification, courses completed, gender, age), would involve further partitions of the state space and that will cause state space disintegration. In order to reduce the state space to a reasonable size, it would be necessary to group sub attributes together, which would result in a much rudimentary model. In modelling a military workforce, it is common to increase the number of attributes to accommodate policy changes and other future needs. Increasing the number of attributes in SD modelling will result in producing many possible states which will lead to a situation where the number of states exceeds the number of individuals in the overall model and this will create a status where the model produces personnel that are 'fractional'. For example, assume the set S represents a current state of a military population that can be partitioned into $l$ states of nonempty subsets (R) corresponding to each rank with numbers corresponding to how many individuals there are within each rank. With the existence of two attributes named TiR and YiS, these rank states will be sub partitioned into $m$ and $n$ nonempty subsets 
(in reality some of these subsets are empty), respectively, as illustrated in Eq. (1). This may result in a situation where the total number of the subsets is more than the number of the personnel within $\mathrm{S}$.

$$
\begin{aligned}
& \mathrm{S}=\bigcup_{i=1}^{l} \mathrm{R}_{i} \text { where } \bigcap_{i=1}^{l} \mathrm{R}_{i}=\varnothing \text { and } \mathrm{R}_{i} \neq \emptyset \text { for } i=1,2, \ldots, l \\
& \mathrm{R}=\bigcup_{j=1}^{m} \mathrm{TiR}_{j} \text { where } \bigcap_{j=1}^{m} \mathrm{TiR}_{j}=\varnothing \text { and } \operatorname{TiR}_{j} \neq \emptyset \text { for } j=1,2, \ldots, m \\
& \mathrm{TiR}=\bigcup_{k=1}^{n} \mathrm{YiS}_{k} \text { where } \bigcap_{k=1}^{n} \mathrm{YiS}_{k}=\varnothing \text { and } \mathrm{YiS}_{k} \neq \emptyset \text { for } k=1,2, \ldots, n
\end{aligned}
$$

Any SD model will follow, mostly, the composite function given in Eq. (2) in distributing stock (representing by s, $a$ and b) to the partitioned states. This results in a situation where the model generates stocks that are fractional, often with a value less than one for many specific states.

$$
\begin{aligned}
& \mathrm{f}: \mathrm{S} \rightarrow \mathrm{R} \text { such that } \mathrm{f}(\mathrm{s})=\eta(\mathrm{S}) / \rho(\mathrm{R}), \forall \mathrm{s} \in \mathrm{S} \\
& \mathrm{g}: \mathrm{R} \rightarrow \mathrm{TiR} \text { such that } \mathrm{g}(a)=\eta(\mathrm{R}) / \rho(\mathrm{TiR}), \forall \mathrm{a} \in \mathrm{R} \\
& \text { h: TiR } \rightarrow \mathrm{YiS} \text { such that } \mathrm{h}(\mathrm{b})=\eta(\mathrm{TiR}) / \rho(\mathrm{YiS}), \forall \mathrm{b} \in \mathrm{TiR} \\
& \text { hogof: } \mathrm{S} \rightarrow \mathrm{YiS} \text { such that } \mathrm{h}(\mathrm{g}(\mathrm{f}(\mathrm{s})))=\eta(\mathrm{S}) /[\rho(\mathrm{R}) \times \rho(\mathrm{TiR}) \times \rho(\mathrm{YiS})], \forall \mathrm{s} \in \mathrm{S}
\end{aligned}
$$

This particular case is most visible at military higher ranks, where there are typically fewer individuals. For instance, in a typical medium-sized army, there may be about 50 BRIG $(\eta(R)=50)$. Once TiR (say 10 values (that is $\rho(R)=10$ ); 0 years, ..., 9 years) and YiS (say 51 values (that is $\rho(Y i S)=51$; 0 years, ..., 50 years) are taken into account, this will result in at least 510 categories into which the stock of 50 individuals is divided to, resulting in fractional numbers of personnel in each category. Taking more attributes into account would aggravate the situation. This fractional representation of people does not give realistic flow patterns. Array vector is well used in Powersim. But because of the difficulties arise in managing and maintaining thousands of array vector's components, the claim to use array vector to accommodate many attributes is absurd.

\subsection{Cumulative error resulted from fraction of personnel}

Another effect of stock-and-flow models typically represents the amount of resources with continuous, non-discrete values (i.e., non integer, rational values), which may result in cumulative errors in data over long time model running periods.

SD models treat the processing being modelled as a whole set (or subset) and the items moving through the simulation are not discrete entities but a cluster of items. In SD workforce modelling, personnel move, as a mob, through the system flowing from one receptacle to another, based on a flow rate for that mob and it is difficult to prevent them from flowing as rational entities (i.e., fractions of personnel). For example, a specified rank-MO group of 25 personnel within it has a separation rate of $11 \%$. If this rate applied to this group, then 2.75 persons will be separated and 22.25 will be retained by the service. This can result in cumulative errors in data over long time model running periods, especially for workgroups consisting of small numbers of individuals. Enhancing a stock-and-flow model to ensure only integer values in each state would essentially be recreating a Monte Carlo model, but in a more complicated and oppressive manner.

The discrete movement of the entities, people in workforce modelling, within DES prevents them from flowing as rational entities. Continuing with the same previous example and by using a DES modelling 
approach, out of these 25 personnel two or three personnel will be separated each model running time. More replications of the model running will make the separation of the three personnel occurring more often than the separation of two personnel and the replications output will try to mimic the $11 \%$ separation rate.

The occurrences of fractional parts are prevented in DES models which will prevent the cumulative errors that may exist in cases of rounding up, down or truncating these fractional parts to maintain an integer. It also makes more sense, by avoiding the fractionalisation of people. The following case study illustrates this in more detail. (This case study is a modification of the workforce example given in Powersim Software AS (2002)).

\subsubsection{Case Study}

Let us assume military recruiting occurs every month to meet a yearly proposed staffing rate. This military recruiting policy assumes that the enlistees inflow in the existing manpower in a rate named change in manpower (m) which is equal to the difference between the preferred manpower level (ml) and the manpower (m) divided by time to adjust manpower (which is set, arbitrarily, to 2). The gap between the preferred manpower level and the actual manpower is halved each month (the time step of the model). Mathematically speaking the inflow will be presented in Eq. (3).

$$
\mathrm{m}=\mathrm{f}(\mathrm{t}, \mathrm{m})=\mathrm{dm} / \mathrm{dt}=(\mathrm{ml}-\mathrm{m}) / 2 \text { where } \mathrm{m}(0)=0
$$

It is elementary to find out that the exact solution of Eq. (3) is $\mathrm{m}=\mathrm{ml}\left(1-\mathrm{e}^{-\frac{1}{2}}\right)$.

The numerical solution of the differential equation $m=f(t, m)$ with an initial condition of $m(a)=0$ and $h=(b-a) / N, t_{0}=a, t_{n}=a+n h, t_{N}=b, n=0,1, \ldots, N-1$, is given by Euler's first degree method (Conte, 1972) as in Eq. (4).

$$
m\left(t_{n+1}\right)=m\left(t_{n}\right)+h m^{\prime}\left(t_{n}\right) \text { with local error of } E=\frac{h}{2} m^{\prime}\left(\tau_{n}\right) \text { where } t_{n} \leq \tau_{n} \leq t_{n+1}
$$

If we assume that there are no promotions, separations, or any other regular activities happening within the simulation period, then a dynamic model is required to find out when and how this yearly preferred staffing level will be satisfied using both DES and SD platforms. For comparison, two simple models; one in the Arena and the other one in Powersim, were developed for this purpose. The integration method used in Powersim was Euler's first order method and the time step for both models was one month. The outputs of these two models for different values of the preferred manpower level are given in Table 1.

It is noticeable that DES-Arena model produced integer results and the SD-Powersim model produced rational results. The fractional parts of the manpower numbers in SD model outputs were rounded to the nearest five digits; otherwise, more time steps are required to reach the integer values of the preferred manpower level. These small rounding errors will accumulate with more activities and attributes added to the model.

In contrast to SD, DES prevents personnel flowing in fractions which will stop the generation of cumulative errors in simulations over long time periods with more activities and attributes added to the model, especially for small workgroups. 
Table 1

The outputs of DES and SD models for different preferred manpower manning level values

\begin{tabular}{|c|c|c|c|c|c|c|}
\hline \multirow[b]{2}{*}{ Time step } & \multirow{2}{*}{$\begin{array}{l}\quad \mathrm{ml}=25 \\
-\mathrm{DES}\end{array}$} & \multicolumn{3}{|c|}{$\mathrm{ml}=50$} & \multicolumn{2}{|c|}{$\mathrm{ml}=100$} \\
\hline & & SD & DES & SD & DES & SD \\
\hline 1 & 12 & 12.50000 & 25 & 25.00000 & 50 & 50.0000 \\
\hline 2 & 18 & 18.75000 & 37 & 37.50000 & 74 & 75.0000 \\
\hline 3 & 21 & 21.87500 & 43 & 43.75000 & 87 & 87.5000 \\
\hline 4 & 23 & 23.43750 & 48 & 46.87500 & 93 & 93.7500 \\
\hline 5 & 24 & 24.21875 & 49 & 48.43750 & 96 & 96.8750 \\
\hline 6 & 25 & 24.60938 & 50 & 49.21875 & 98 & 98.4375 \\
\hline 7 & & 24.80469 & & 49.60938 & 99 & 99.21875 \\
\hline 8 & & 24.90234 & & 49.80469 & 100 & 99.60938 \\
\hline 9 & & 24.95117 & & 49.90234 & & 99.80469 \\
\hline 10 & & 24.97559 & & 49.95117 & & 99.90234 \\
\hline 11 & & 24.98779 & & 49.97559 & & 99.95117 \\
\hline 12 & & 24.99390 & & 49.98779 & & 99.97559 \\
\hline 13 & & 24.99695 & & 49.99390 & & 99.98779 \\
\hline 14 & & 24.99847 & & 49.99695 & & 99.9939 \\
\hline 15 & & 24.99924 & & 49.99847 & & 99.99695 \\
\hline 16 & & 24.99962 & & 49.99924 & & 99.99847 \\
\hline 17 & & 24.99981 & & 49.99962 & & 99.99924 \\
\hline 18 & & 24.99990 & & 49.99981 & & 99.99962 \\
\hline 19 & & 24.99995 & & 49.99990 & & 99.99981 \\
\hline 20 & & 24.99998 & & 49.99995 & & 99.9999 \\
\hline 21 & & 24.99999 & & 49.99998 & & 99.99995 \\
\hline 22 & & 24.99999 & & 49.99999 & & 99.99998 \\
\hline 23 & & 25.00000 & & 49.99999 & & 99.99999 \\
\hline 24 & & & & 50.00000 & & 99.99999 \\
\hline 25 & & & & & & 100.00000 \\
\hline
\end{tabular}

\section{Conclusion}

Any operations research technique may be applied in workforce modelling according to the problem under investigation, nevertheless DES and SD approaches seem to be the most appealing applications in the area of modelling military workforces which include the individuals' interaction within the system.

Many military organisations require their workforce models to be able to accommodate many new attributes to adopt any future organisation policy changes. DES enables attributes associated with each entity to be readily monitored, and the computation associated with this attribute tracking does not increase with the larger numbers of attributes. In SD models, attribute tracking becomes harder and increasingly more complex with higher numbers of attributes.

DES works at an individual level, allowing full representation of each individual's history and the interaction between specific individuals. Unlike SD, DES prevents fractional personnel flow, which serves to prevent the generation of cumulative errors in simulations over long time periods, especially for small workgroups. Therefore, we believe DES is the optimal solution to address most of the issues facing military workforce modelling.

\section{References}

Barton, P., Bryan, S., \& Robinson, S. (2004). Modelling in the economic evaluation of health care: selecting the appropriate approach. Journal of Health Services Research \& Policy, 9(2), 110-118.

Brailsford, S. C., Desai, S. M., \& Viana, J. (2010, December). Towards the holy grail: combining system dynamics and discrete-event simulation in healthcare. In Simulation Conference (WSC), Proceedings of the 2010 Winter (pp. 2293-2303). IEEE. 
Chahal, K., \& Eldabi, T. (2010). A multi-perspective comparison for selection between system dynamics and discrete event simulation. International Journal of Business Information Systems, 6(1), 4-17.

Conte, D. (1972). Elementary Numerical Analysis. 3nd Edn., McGraw-Hill, New York.

Forrester, J.W. (1961). Industrial Dynamics. 1st Edn., Pegasus Communications, Waltham.

Gordon, G. (1961). A general purpose system simulation program. Proceedings of the Eastern Joint Computer Conference: Computers - Key to Total Systems Control, (TSC61), ACM New York, NY, USA.

Hollocks, B. W. (2006). Forty years of discrete-event simulation-a personal reflection. Journal of the Operational Research Society, 57(12), 1383-1399.

Kelton, W.D., Sadowski R.P., \& Stuttock D.T. (2007). Simulation with Arena. $5^{\text {th }}$ ed., McGraw-Hill Education, Boston, USA.

Tanha, M., Torshizi, S. D., \& Shamala, S. (2012). A discrete event simulator for extensive defense mechanism for denial of service attacks analysis. American Journal of Applied Sciences, 9(6), 909916.

Morecroft, J., \& Robinson S. (2005). Explaining puzzling dynamics: Comparing the use of system dynamics and discrete-event simulation. Proceedings of the 23rd International Conference of the System Dynamics Society, The System Dynamics Society, Boston, USA.

Morecroft, J., \& Robinson, S. (2006). Comparing discrete event simulation and system dynamics: modelling a fishery. Proceedings of the 2006 OR Society Simulation Workshop SW06, Leanington, Spa, UK, pp:137-148.

Powersim, A.S., \& Bergen, N. (2002). http://www.powersim.com. Powersim studio-Help Psmain.chm::/PsEngine/Working with Variable Definitions.

Raczynski, S. (2012). Semi-discrete events and models in categorical language. International Journal of Simulation Modelling, 11(2), 89-96.

Robinson, S. (2005). Discrete-event simulation: from the pioneers to the present, what next?. Journal of the Operational Research Society, 56(6), 619-629.

Shannon, R. E. (1975). Simulation: a survey with research suggestions. AIIE Transactions, 7(3), 289301.

Sweetser, A. (1999, July). A comparison of system dynamics (SD) and discrete event simulation (DES). In 17th International Conference of the System Dynamics Society (pp. 20-23).

Tako, A. A., \& Robinson, S. (2009). Comparing discrete-event simulation and system dynamics: users' perceptions. Journal of the operational research society, 60(3), 296-312.

Tako, A. A., \& Robinson, S. (2010). Model development in discrete-event simulation and system dynamics: An empirical study of expert modellers. European Journal of Operational Research, 207(2), 784-794. 